EPJ Web of Conferences 13, 04003 (2011)

DOI: $10.1051 /$ epjconf/20111304003

(c) Owned by the authors, published by EDP Sciences, 2011

\title{
The Modification of the Scalar Field in dense Nuclear Matter
}

\author{
Jacek Rożynek, a
}

The Andrzej Sołtan Institute for Nuclear Studies, Hoża 69, 00681 Warsaw, Poland

\begin{abstract}
We show the possible evolution of the nuclear deep inelastic structure function with nuclear density $\rho$. The nucleon deep inelastic structure function represents distribution of quarks as function of Björken variable $x$ which measures the longitudinal fraction of momentum carried by them during the Deep Inelastic Scattering (DIS) of electrons on nuclear targets. Starting with small density and negative pressure in Nuclear Matter (NM) we have relatively large inter-nucleon distances and increasing role of nuclear interaction mediated by virtual mesons. When the density approaches the saturation point, $\rho=\rho_{0}$, we have no longer separate mesons and nucleons but eventually modified nucleon Structure Function (SF) in medium. The ratio of nuclear to nucleon SF measured at saturation point is well known as "EMC effect". For larger density, $\rho>\rho_{0}$, when the localization of quarks is smaller then $0.3 \mathrm{fm}$, the nucleons overlap. We argue that nucleon mass should start to decrease in order to satisfy the Momentum Sum Rule (MSR) of DIS. These modifications of the nucleon Structure Function (SF) are calculated in the frame of the nuclear Relativistic Mean Field (RMF) convolution model. The correction to the Fermi energy from term proportional to the pressure is very important and its inclusion modifies the Equation of State (EoS) for nuclear matter.
\end{abstract}

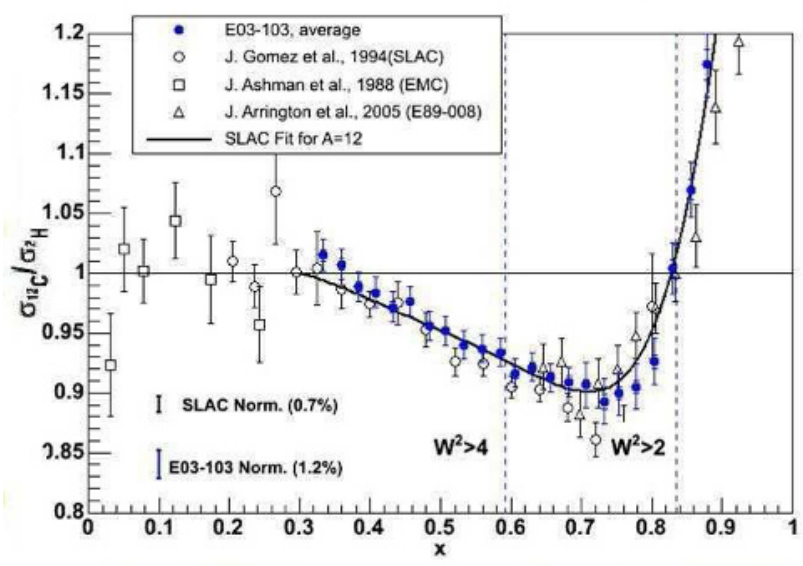

Fig. 1. EMC effect in the electron scattering on nuclear targets. Ratio of the two $e-A$ cross-sections $\left(C_{12}\right.$ to $\left.D\right)$.

\section{Introduction}

The observed enhancement of the nuclear to deuterium cross sections ratio for Björken variable $x \sim 0.6$ known as EMC effect is still the open subject. The main issue of this work is the explanation of nuclear medium modifications in the deep inelastic process on nuclear targets caused by nucleon-nucleon interactions and nucleon motion inside nucleus. The last measurements [1] confirm quite strong nuclear dependence, as witnessed by Fig. 1 (where it is shown for small mass number $\mathrm{A}=12$ ), of the cross section ratio on nuclei to deuteron. This ratio, in this work denoted by $R(x)$, almost does not depend on $Q^{2}$ for $x>0.02$ (their dependence are cancelled in the ratio). This is strong evidence for the influence of nuclear field on the partonic structure of nucleons. New experiments are going to explore large $x$ region where both nucleon in nucleus and parton in nucleon are strongly correlated.

DIS on nuclei[2] - the main idea and our framework of the nuclear SF are presented in section (2). In the wide range of nuclear density $0<\varrho<3 \varrho_{0}$ to nucleons interact by meson exchanges. Therefore in addition to the electronnucleon scattering the scattering on exchanged mesons also take place and contribute in the sea region populated mainly by quark-antiquark $q \bar{q}$ pairs. Our model for parton distribution in nuclei is based on the assumption that nuclear interaction is switch off for big values of $x$ and consequently in this regime, the collective motion of partons in nuclear medium take place. The sea Parton Distributions (PD) are described by allowing for additional virtual pions in hadron to appear (we call them "nuclear pions") in quantity which reproduces both the nuclear lepton pair production data and saturates the energy-momentum

\footnotetext{
${ }^{a}$ e-mail: rozynek@fuw.edu.pl
}

This is an Open Access article distributed under the terms of the Creative Commons Attribution-Noncommercial License 3.0, which permits unrestricted use, distribution, and reproduction in any noncommercial medium, provided the original work is properly cited. 
sum rule. Good agreement with the experimental data has been obtained [3]. The quark localization is proportional to $1 / x$ and this relation introduces dependence of the nucleon structure function on nuclear medium. In DIS in nuclear medium, part of pions contribute to the sea quark structure of nucleon, but for small $x<0.01$ the uncertainty in the life time of intermediate photon-quark state becomes comparable (or larger) then the mean distance between nucleons what results in the shadowing of single nucleons contributions. These medium effects, namely the changes in the nucleon rest energy and the enhancement of the sea quark contribution (as simulated by "nuclear pions") modify the transverse parton momentum distribution inside NM. It was shown that in order to reproduce the observed behavior of experimental data and describe the $x$ dependence one has to determine the amount of the additional intermediate pions (mediating the nucleon-nucleon interaction [4]) in the function of Björken $x$. In particular, the magnitude of nuclear Fermi motion is sensitive to the residual interactions between partons, influencing both the nucleon structure function and the value of the nucleon mass in nuclear matter [5]. In the section (4) we will discuss time the EMC effect in function of nuclear density. The influence of these modifications to the Equation of State (EOS) in NM will be discussed in the last section. Good agreement predictions with experiments in heavy ion collisions have been obtained [6].

\section{The nuclear deeply inelastic limit}

The Björken variable $x$ is the fraction of momentum $k$ carried by quark in the nucleon and is equal in this model to the ratio of longitudinal momenta $x_{A, N}=\left(k_{o}+k_{3}\right) / M A, N$. It was shown that in fact the nuclear SF in CM depends on the contributions from both scalar $U_{S}$ and vector $U_{V}$ potentials. The nuclear quark $\mathrm{SF} F_{2}^{A}$ in a nucleus with the mass number $A$ is constructed in the convolution model (CM) from the free nucleon $\mathrm{SF}_{2}^{N}\left(x \equiv x_{N}\right)$ in the nucleon and the nucleon distribution function $\rho^{A}\left(y_{A}\right)$ in the nucleus. The general convolution formula in the relativistic Fermi gas model [7] for nucleon $\mathrm{SF}_{2}^{A}$ is:

$\frac{1}{A} F_{2}^{A}\left(x_{A}\right)=\int d y_{A} \rho^{A}\left(y_{A}\right) F_{2}^{N}\left(x_{A} / y_{A}\right)+\int d y_{\pi} \rho_{\pi}^{A}\left(y_{\pi}\right) F_{2}^{\pi}\left(x_{A} / y_{\pi}\right)$,

where $F_{2}^{\pi}$ and $F_{2}^{N}(x)\left(F_{2}^{N}(x)\right)$ are the parton distributions in the free, or medium modified, pion and in the free (or bound) nucleon respectively, $\rho_{\pi}^{A}$ is the probability distribution for nuclear pions in the nuclei and the nuclear density $\rho^{A}$ is given by:

$\rho^{A}\left(y_{A}\right)=\frac{4}{\rho} \int \frac{d^{4} p}{(2 \pi)^{4}} S_{N}\left(p^{o}, \mathbf{p}\right)\left(1+p_{3} / E^{*}(p)\right) \delta\left(y-\left(p_{o}+p_{3}\right) / \mu\right)$

where $p=\left(p_{o}, \mathbf{p}\right)$ is the nucleon four momentum, $E^{*}=$ $\sqrt{\mathbf{p}^{2}+\left(m-U_{S}\right)^{2}}$ and the factor $\left(1+p_{3} / E^{*}\right)$ corrects[8] the nonrelativistic expression. The nucleon energy is equal to the chemical potential $p_{o}=\mu$ at the Fermi surface -
$E_{F} \equiv \mu=\sqrt{\mathbf{p F}^{2}+\left(m-U_{S}\right)^{2}}+U_{V}$ (Hugenholtz-van Hove theorem). Here the nucleon spectral function was taken in the RMF approach: $S_{N}=n(p) \delta\left(p^{o}-\left(E^{*}+U_{V}\right)\right)$.

In the nuclear medium, characterized by $E / A$ and Fermi energy $E_{F}$, the rest energy of the nucleon $M_{B}=\sum_{i} k_{N i}^{+}$, in the Björken on shell limit (short interaction times), takes effectively different value (for large $x$ ) than its free nucleon mass $M$ and gives the proper description[3] of EMC data in Fig. 1. It can be thought as the sum of the corresponding partonic energies $k_{N i}^{0}$ expressed in the rest frame of nucleon (notice that they differ from $k_{A i}^{0}$ ). Such $M_{B}$, accounts therefore effectively for the Fermi motion of nucleons inside the nucleus. This is, in addition to the standard Fermi smearing on a nuclear level, the influence of the Fermi motion emerging from a nucleonic $(x)$ level.

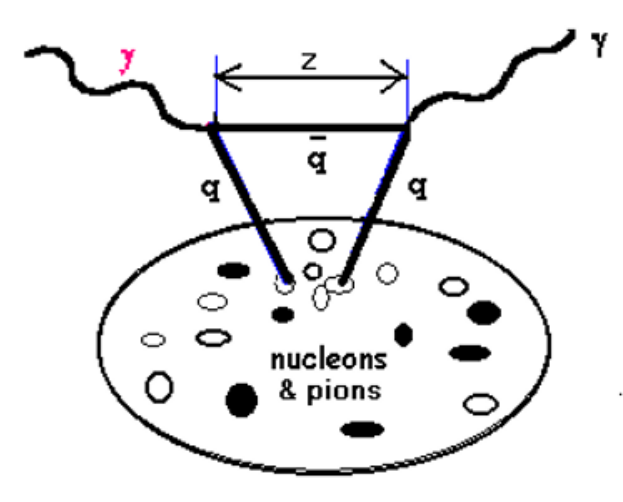

Fig. 2. Struck quark $q$ from pion or nucleon create the (anti) quark $\bar{q}$ which propagates through the nucleus by the distance $z \sim 1 / M x$.

The nucleon distribution is simplified in the RMF in saturation point (no pressure) to the form[7]:

$$
\rho^{A}\left(y_{A}\right)=\frac{3}{4 v_{A}^{3}}\left(v_{A}^{2}-\left(y_{A}-1\right)^{2}\right)
$$

where $v_{A}=p_{F} / \mu$, and $y$ takes the values given by inequality $\left(1-p_{F} / \mu\right)<y<\left(1+p_{F} / \mu\right)$. Thus all the nuclear dependence is hidden in nucleon chemical potential $\mu=E / A=E_{F}$ when the pressure is absent at the saturation point $[7,9,3]$. However for finite pressure the situation is different[10].

\section{Finite density - $A$ dependence}

Generally the A dependence of the EMC effect is strong only for small $x<0.1$. Is rather weak for intermediate $x$ and for large $x>0.9$ might depend on NN correlations which also depend on $A$.

The intermediate mesons contribute to the behavior at small $x$. At small values of the Björken variable $x(<.05$ i.e., in the shadowing region), the SF $F_{2}$ turns out to be smaller in nuclei then in free nucleons. There are several 


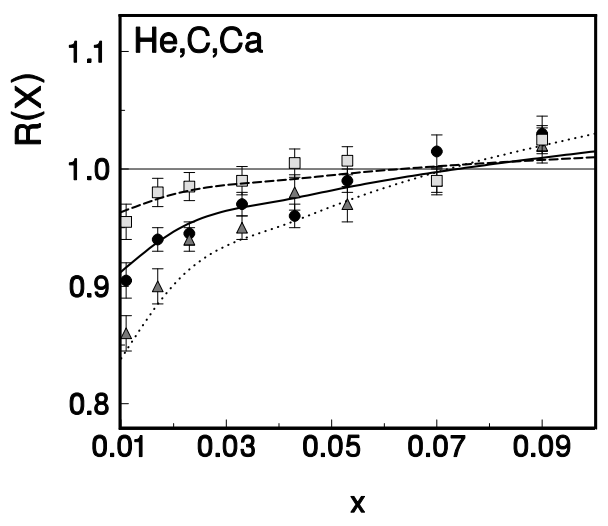

Fig. 3. The EMC ratio for $\mathrm{He}$ (squares, dashed line), C(circles, solid line), Ca(triangles, dots). Data are from [14].

descriptions of this phenomenon. Assuming that incoming photon splits into $q \bar{q}$ before reaching nucleus then we have absorption of this pairs which depends on $x$. This can be related to multiple scattering and diffraction [11]. Here we will model the shadowing in the frame of partonic recombination approach $[12,13]$. In the shadowing we have two distinguishing points. For mean free path of quarks $z_{S}(x)$ larger then intermediate distance between nucleons, $z_{S}(x)<r_{N}$, the nucleons are not longer localized by photons as belonging to particular nucleon and shadowing starts. For $r_{N} \simeq 2 f m$ we have $x_{S} \simeq 0.1$. The shadowing should saturate at $x_{f} \simeq 0.01$ when $z>2 r_{0} A^{1 / 3}$ and the resolution is larger then nuclear diameter. Finally shadowing should depend on the distance $z-z_{S}$ and for saturation distance the depletion of the cross section should be proportional to the number of shadowing nucleons in the nucleus - $\left(A^{1 / 3}-1\right),\left(A^{1 / 3}\right.$ because the shadowing take place along $z$ direction only). Summarizing we show our parametrization of the cross section in the region $0.01<x<0.1$ by the following function:

$$
R(x)=1-K \sqrt{\left(z-z_{s}\right)}\left(A^{1 / 3}-1\right)
$$

on Fig. 3 for Helium, Carbon and Calcium. Above formula gives reasonable fit to data with only one free parameter which shows that the nuclear dependance of shadowing region is described quite reasonable.

For intermediate region of $x$ calculations were made in the local density approximation where the Fermi momentum $p_{F}=\left(3 / 2 \rho \pi^{2}\right)^{1 / 3}$ was calculated from the local nuclear matter density $\rho$. For C, Ca and Ag we choose respectively $k_{F}=220,240,260 \mathrm{MeV}$ respectively [15]. The distribution parameters above the Fermi level: the end of the distribution tail $p_{T}$ or exponent $r$ were calculated from the normalization condition for $n(p)$. In finite nuclei the nucleons partially occupy their single particle states below the Fermi level with the long distance tail above [16]; therefore the momentum distribution function $n(p)$ was model by:
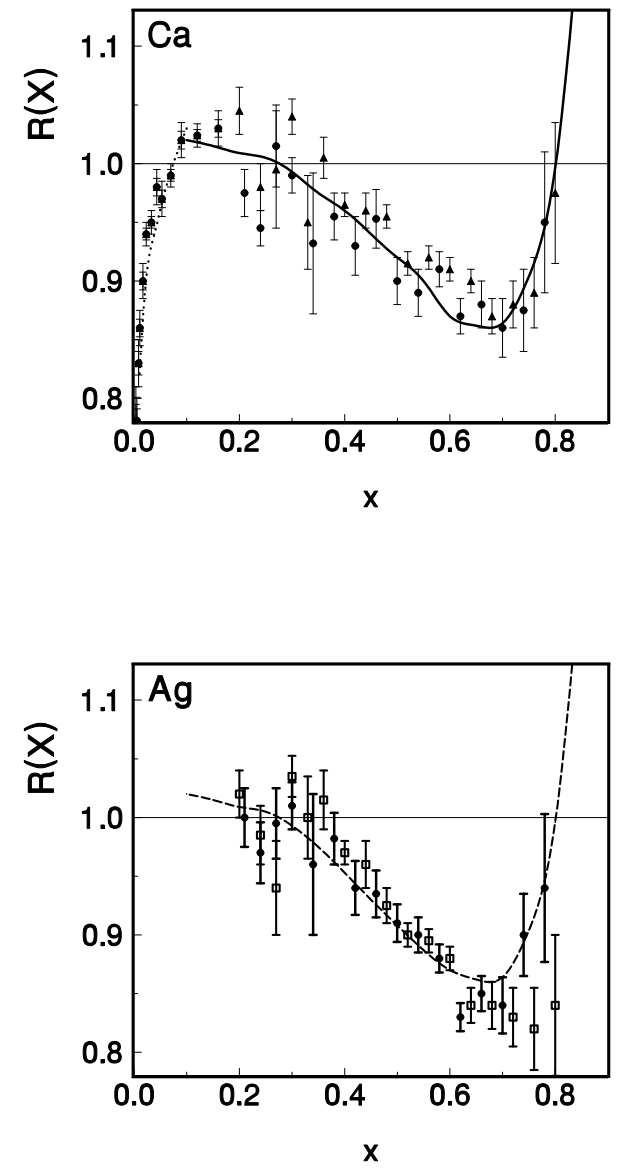

Fig. 4. $\mathrm{R}(\mathrm{x})$ - the EMC ratio for Calcium (upper) and Lead (bottom) to deuterium.

$$
\begin{array}{cc}
n(p)=n_{0} & \text { for } p \leq p_{F} \\
n(p)=n_{0} e^{\left(p-p_{F}\right)^{r}} & \text { for } p>p_{F} .
\end{array}
$$

The dependence from the details of the distribution tail above the Fermi surface was found to be negligible in the presented approach for that range of $x$. We compare it also with density distributions coming the standard nuclear harmonic oscillator potential for light nuclei with $A$ dependent phenomenological parameter $h \omega=45 A^{-1 / 3}+25 A^{-2 / 3}$ [17].

In our model the new sea parton distributions described by the modified cloud of virtual pions is the response for the depletion of cross section for intermediate $x$ (which is described by the change of the mass distribution as a function of $x$ due to the final state interaction). Both effect lead to the conservation of nuclear single particle momentum sum rule. This is very important. Our description of theses features are in good agreement with experimental data: the EMC effect and nuclear lepton pair production data has been described essentialy without free parameters. 


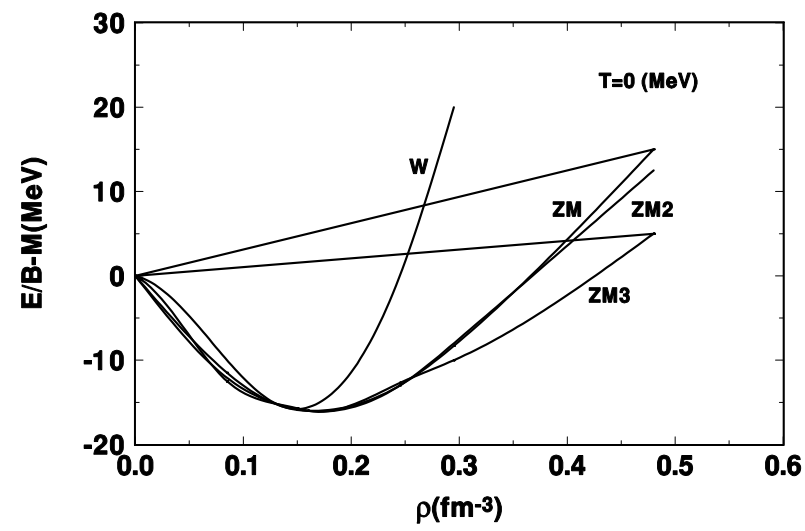

Fig. 5. The nucleon energy in function of nuclear matter density in the RMF approach for Walecka and ZM models.

\section{RMF models, EOS and parton SF}

For non zero pressure in nuclear matter the Fermi energy is not equal to the average binding energy but there are the well known correction (see for example)[18]:

$$
\begin{array}{r}
E_{F}=\frac{d}{d \varrho}\left(\frac{E}{\Omega}\right) \\
E_{F}=\left(\frac{E}{A}\right)+\varrho \frac{d}{d \varrho}\left(\frac{E}{A}\right)
\end{array}
$$

where $A / \varrho=\Omega$ give volume. Equivalently we can write with $p=-\left(\frac{\partial E}{\partial \Omega}\right)_{B}=\varrho^{2} \frac{d}{d / \varrho}\left(\frac{E}{A}\right)$ :

$$
E_{F}=E / A+p / \varrho \equiv E / A+E_{\text {press }}
$$

The $E_{\text {press }}$ is generated by the nuclear interaction via meson exchange and for low pressure below saturation point is negative and is represented basically by the scattering on virtual meson, see Fig. 2. These contributions proportional to the pressure are greatest for density $\varrho=\varrho_{0} / 2$ where the density gradient is large. For bigger density this contributions became smaller and vanish in saturation point. For increasing pressure nucleons begin to overlap. There are no place for contribution from exchanged meson but rather the nucleon SF which is built from meson cloud should be modified. Consequently in order to satisfy MSR[3] in (DIS) the nucleon SF in the nuclear medium has to be changed. For region $\varrho \geq \varrho_{0}$ the nucleon SF begin to change into bound nucleon SF which like nucleon SF has no explicit meson degree freedom at saturation density. The Equation of State (EOS) for nuclear matter has to match the saturation point with compressibility $K^{-1}=9 \varrho^{2} \frac{d^{2}}{d \varrho^{2}} \frac{E}{A}$ but then the behavior for higher densities is different for different RMF models, see Fig. 5. We present here two extreme examples: stiff model of Walecka [20] (with $K^{-1}=450$

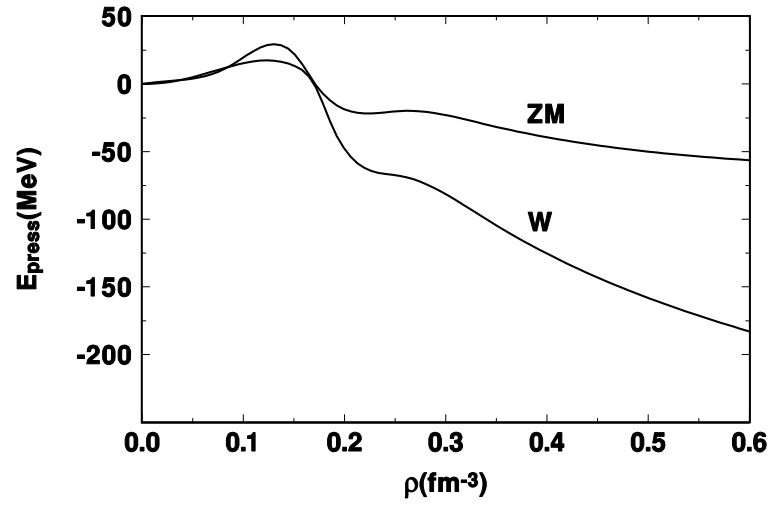

Fig. 6. $\mathrm{R}(\mathrm{x})$ - The density dependent energy $E_{\text {press }}$ inside nuclear matter for stiff (Walecka) and soft (ZM) EoS.

$\mathrm{MeV}$ ) and two soft Zimanyi-Moszkowski [21] non linearmodels [22] with $K^{-1} \simeq 200 \mathrm{MeV}$, close to the experimental estimate. ${ }^{1}$ The density dependent energy $E_{\text {press }}$ carried by meson field is shown in Fig. 6. The role of this term in comparison to $E / A$ (previous picture) is strong for stiff EOS.

Calculation of the nuclear SF for positive pressure have to include the changes in Fermi energy Eq.(4) along with the finite pressure [10]. It increases $E_{F}$ by $8 \%$ for density $3 \rho_{0}$. The second sum rule is sensitive for these finite pressure correction $E_{\text {press }}=E_{F}-E / A$ as can be seen from the integral taken in limits $\left(E_{F}-p_{F}\right)<A y_{A} / E<\left(E_{F}+p_{F}\right)$ :

$$
\frac{A \int d y_{A} y_{A} \rho^{A}\left(y_{A}\right)}{E}=1+\frac{A E_{\text {press }}}{E}
$$

The integral depends only from the Fermi energy which additionally determine the onset of the maximum for nuclear density $\rho^{A}$. We see that second the sum rule is broken by the factor $E_{F} / \mu$. Therefore we have to change the quark SF. Scaling the Björken $x$ by $E_{F} / \mu$ will restore the MSR:

$$
\begin{aligned}
\frac{1}{A} \int F_{2}^{A}\left(x_{A}\right) d x_{A} & =\int d y_{A}\left(y_{A}\right) \rho^{A}\left(y_{A}\right) \int F_{2}^{N}\left(\frac{E_{F}}{\mu} x_{A}\right) d x_{A} \\
& =\left(M_{A} / M_{N}\right) \int F_{2}^{N}\left(x_{N}\right) d x_{N}
\end{aligned}
$$

Such a scaling of $x_{N}=\left(M_{A} / M_{N}\right) x_{A}$ by definition will reduce the nucleon mass $M$ by factor $\mu / E_{F}$. We just demonstrate how the change of the quark structure function can help us on the nuclear level. However not only momentum sum rule is satisfied. These finite pressure correction to the

\footnotetext{
${ }^{1}$ Biro and Zimanyi [23] proposed a new effective Lagrangian, adding to the usual ZM-Lagrangian a tensor coupling analogous to the one which leads to the anomalous gyromagnetic ratio. An additional free parameter in this term is suggested to be eliminated in favor of the improvement of the spin-orbit splitting for finite nuclei calculations.
} 


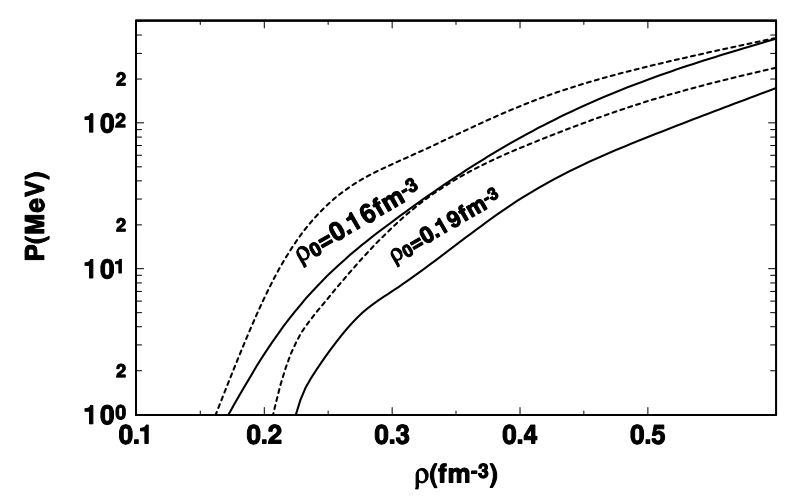

Fig. 7. Nuclear pressure in function of density for the most frequent pure scalar-vector models: version[19] for $\rho_{0}=.19 \mathrm{fm}^{-3}$ $\left(C_{v}{ }^{2}=195.9, C_{s}{ }^{2}=267.1\right)$ and version[20] for $\rho_{0}=.16 \mathrm{fm}^{-3}$ $\left(C_{v}{ }^{2}=273.8, C_{s}^{2}=357.4\right)$ are denoted by dotted and our modified versions by solid lines.

nucleon mass make the EoS much softer. These main results displayed in Fig. 7 were obtained by solving numerically equations for energy and pressure similar to the ordinary Walecka model but with the modified nuclear mass given by $M\left(\mu / E_{F}\right)=M /\left(1+E_{\text {press }} / \mu\right)$ which has the extra dependence from energy and and its density derivative (pressure). We see that these correction applied to two standard sets of parameters in Walecka model make this model softer with the good or at least much better agreement with semi experimental estimate[24] from heavy ion collision. The EOS obtained with $\rho_{0}=.19 \mathrm{fm}^{-3}\left(C_{v}{ }^{2}=195.9, C_{s}{ }^{2}=\right.$ 267.1 ) coincides with the allowed course of EoS (restricted area in [24] obtained using analyses which extract from matter flow in heavy ion collision the high pressure obtained there).

\section{Summary}

Our model calculations performed in the frame of relativistic mean field approach shows how important are the modifications (particularly for those RMF with stiff EoS) of nucleon mass which restore the momentum sum rule within the parton structure in nuclear matter. The presented analysis of the nuclear distribution function in the Björken limit ask to modify the nucleon mass only above the saturation point, the standard nuclear physics remains unchanged. In the presented work we assumed that nucleon structure function above saturation point has the same form as in the equilibrium of nuclear matter.

\section{Acknowledgements}

Partial supports of the Ministry of Science and Higher Education under the Research Project No. N N202046237 for is acknowledged.

\section{References}

1. J. Arrington et. al, prep nucl-ex/0701017, Phys. Rev. C73 (2006) 035205.

2. S.V. Akulinichev, et al. Phys. Lett.158B (1985) 485; Phys. Rev. Lett. 55 (1985) 2239; G.V. Dunne and A.W. Thomas, Phys. Rev. D33 (1986) 2061; Nucl.Phys. A455 (1986) 701; R.P. Bickerstaff, M.C. Birse and G.A. Miller, Phys. Rev. C D33 (1986) 3228; J. Rozynek, Int. J. Phys. E9, (2000)195.

3. J. Rożynek, G.Wilk, Phys. Rev. C71 (2005) 068202.

4. J. Rożynek, G.Wilk, Acta Phys. Pol. B35 (2004) 2303.

5. J. Rożynek, Nucl. Phys. A755 (2004) 357c.

6. J. Rożynek, Acta Phys. Pol. B37 (2006) 95.

7. M. Birse, Phys. Lett, B299, (1993) 188.

8. L.L. Frankfurt and M.I. Strickman, Phys. Lett. 183B (1987) 254.

9. J. R. Smith and G. A. Miller, Phys. Rev. C65 (2002) 055206.

10. J. Rożynek, Acta Phys. Pol. B39 (2008) 343.

11. N. Armesto et al. Eur. Phys. J. C29 (2003) 531.

12. N. N. Nikolaev, V.I.Zakharov, Phys. Lett. 55B, 397 (1975).

13. M. Arneodo Phys. Rep. 240 (1994) 301.

14. P. Amaudruz et al, Z. phys. C52 (1991) 387; M. Arneodo et al. Nucl. Phys. B441 (1995) 12.

15. A. Monitz et. al., Phys. Rev. Lett. 26 (1971) 445.

16. V.R. Pandharipande, C.N. Papanicolas, and J. Wambach, Phys. Rev. Nucl.Part.Sci.37, (1988) 133; Mahaux and R. Sartor, Nucl.Phys. A503 (1989) 525; J. Piekarewicz, R.A. Rego, Phys. Rev. C45, (1992) 1654.

17. B.L. Birbrair, A.M. Levin, A.G. Shuvaev, Nucl. Phys. A496 (1989) 704.

18. K. Kumar, "Perturbation Theory and the Many Body Problem", North Holland, Amsterdam 1962 (1995).

19. B. D. Serot and J. D. Walecka, Adv. Nucl. Phys. Vol. 16 (Plenum, N. Y. 1986).

20. Furnstahl and B. D. Serot, Phys. Rev. C41 262 (1990).

21. A. Delfino, C.T. Coelho and M. Malheiro, Phys. Rev. C51 (1995) 2188

22. J. Zimanyi and S.A. Moszkowski, Phys. Rev. C42 (1990) 1416; Phys. Rev. C44 (1990) 178.

23. T. S. Biró and J. Zimanyi, Phys. Lett. B391, 1 (1997)

24. P. Danielewicz, R. Lacey, W. G. Lynch, Science 298 (2002) 1592. 